\title{
MORALISCHE INTEGRATION. ÜBER EINE LÜCKE IN HEGELS THEORIEBILDUNG UND DIE NIETZSCHEANISCHEN VERSUCHUNGEN DER ANTWORT
}

\section{Gesellschaftlich-politische Wirklichkeit, das Denken über die erfahrene Wirklichkeit, und der Wandel der Wirklichkeiten}

Unter den großen Deutungen, die die verschlungenen Wege der Philosophie vom Deutschen Idealismus bis in die eigene Gegenwart der Erfahrungen des 20. Jahrhunderts nachzuzeichnen und in ein strukturiertes Bild zu bringen versuchen, ist die von Karl Löwith vielleicht die berühmteste geworden. Löwith hatte einst an der Polarität von Hegel und Nietzsche den - so der Untertitel seines bekannten Buches - »revolutionären Bruch im Denken des 19. Jahrhunderts « festgemacht: jenen großen Umbruch, in dessen Auswirkungen alles Weitere, bis heute, steht. Hegel stand darin als die letzte Kulmination der klassischen Tradition, und Nietzsche als der Beginn jenes Denkens, das das ganze folgende Jahrhundert, das zwanzigste, umtreiben sollte. In Nietzsche, und den Vermittlungsgestalten, die diesen Epochenbruch vorbereitet haben, erkannte Löwith die Geburt des wahrhaft modernen - und zuletzt heute spostmodern genannten - geistigen Zeitalters.'

Nietzsche tritt hier auf die Seite von Marx, wird zum Bruder von Marx: als die Ideologiekritik an den Erbschaften der bürgerlichen Epoche. - Das Verhältnis von Hegel und Marx ist seit Dezennien, im Grunde seit Beginn des 20. Jhs. und zumal im Zusammenhang der sich formierenden Sozialwissenschaften, breit diskutiert, auch gegeneinander abgewogen. Die Bezüge zwischen Hegel und Nietzsche dagegen sind noch wenig verstanden. Auch im Gefolge Löwiths blieb es, über die gleichsam physiognomische Polarisierung der $>$ Mentalitäten beider hinaus, bei entweder einem vermittlungslosen Sich-nichtrecht-entscheiden-Können ${ }^{2}$ oder bei wechselseitigen Verteufelungen beider Anhängerschaften. Dabei sind Hegel und Nietzsche die zwei Seiten desselben, zwei Seiten der denkerischen Bewältigung einer Erfahrung der Prozesse der Moderne.

Hegel hat die moderne (’protestantische`) Subjektivität und ihr Selbstverständnis ihrer Freiheit zur Grundlage gemacht - und scheint zugleich keine Räume des Nicht-Integrierten zu kennen. Das sollte immer wieder die Hegel-skeptischen Programme, den antihegelschen Affekt auf den Plan rufen. ${ }^{3}$ Ich möchte deshalb im folgenden gezielt noch einmal Nietzsche, als die große Gegenströmung, die das letzte Viertel des 19. Jhs. ausgebildet hat, in den Blick heben, den Bezug jedoch von der anderen Seite her beleuchten. Erklärt werden muß: warum wurde Nietzsche möglich?, konnte der Nietzscheanismus so große Resonanz finden und gerade auch was das gesellschaftliche und politische Denken betrifft? - Meine These ist, daß die bekannt prekäre Position, die der moralischen Reflexion in Hegels praktischer und im speziellen politischer Philosophie zugemessen wird, das Einfallstor für die skulturkritischen Umdeutungen des Gesellschaftlichen wurde, welche das Denken des 20. Jhs. prägen. Diese kristallisieren sich in

1 Karl LÖWITH, Von Hegel zu Nietzsche. Der revolutionäre Bruch im Denken des 19. Jahrhunderts, Zürich / New York 1941. Löwith hatte dies an den Veränderungen im Verständnis der bürgerlichen Gesellschaft, der Arbeit, der Bildung, der Humanität und der Christlichkeit festgemacht.

2 So auch Löwith selbst.

3 J. Habermas hat das Löwithsche Thema, unter rationalitätstheoretischer Neuformulierung, noch einmal aufgenommen. Doch hat er im Grunde den Denkrahmen Löwiths nicht wirklich verlassen. Vgl. J. HABERMAS, Der philosophische Diskurs der Moderne, Frankfurt/M. 1985, 104-129 (»Eintritt in die Postmoderne: Nietzsche als Drehscheibe«), 144-165. 
den verschiedenen Nietzscheanischen Motivationen und Denkstrukturen des neueren Sozial- und politischen Denkens.

Die These ist eingebettet in ein Dreifaches von Rahmenüberlegungen, die im Folgenden nur angerissen werden können, jedoch nicht mehr in die eigentliche Thematisierung dieses Aufsatzes fallen: Was einst später Schelling, Feuerbach, Kierkegaard als Kritik an Hegel und dem ganzen in Hegel kulminierenden Erbe formuliert haben, hat in Nietzsche seine die heutige Philosophie prägende Transformation, auch Provokation, gefunden; was sich als Kritik bezüglich der Subjektivität des (spekulativen) Denkens - und thematisch: vor allem Religionsphilosophie - artikuliert hatte, hat sich in den verschiedenen Nietzscheanischen Motivationen und Denkstrukturen zu einem prinzipiell transklassischen Denken der Reflexion der Erfahrungen der Moderne gestaltet. Wenn die Seite der Anfechtungen in den Blick gebracht werden soll - und theoriekonzeptionell in ihrem berechtigten Gehalt eingebracht -, so sollte darum von Hegel aus dieser Gegner, und Nietzsche als die Spitze jener Anfechtungen, nicht zu klein gemacht werden. Auch gibt es, zweitens, durchaus eine Linie der Verbindung. Das nachhegelsche Denken läßt sich als die Radikalisierung einer - jeweils richtungsbetreffenden - Sachdimension, was bei Hegel überhaupt erst als Problem eröffnet, als Wirklichkeit und Frage benannt worden ist, begreifen. Nietzsche etwa ist auch und vor allem eine radikalisierte Antwort auf die durch Hegel in das Denken eingeführte Sachwirklichkeit `Gesellschaft, und Erfahrung 'gesellschaftlicher` Mittelbarkeit des Menschlichen. Die Rhetorik von Bruch, Umwälzung usw. verstellt da leicht den essentiellen denkgeschichtlichen Zusammenhang. Und drittens ist vieles, bes. das Laute, des Nietzscheanismus der Epoche vornehmlich 'zeitgeistige r mentale Reaktion oder Reflex. Andererseits aber ist nicht wenig des dauerhaft gewirkt habenden Nietzscheanismus, Nietzscheanischer Einflüsse, kein Gegensatz zu Hegel, sondern im Grunde Gestalt von dessen Weiterführung. Diese zu sehende Weiterführung, so die Rahmenüberlegung, ist Weiterführung auch angesichts einer weiterentwickelten (gesellschaftlichen) Wirklichkeit, antwortet, und durchaus in Hegel integrierbar, auf neue, verschärfte Wirklichkeiten. Der heutige Stand dieser Wirklichkeiten hat dabei Strukturgegebenheiten erreicht - geschaffen - , die nicht mehr Potentialen Nietzschescher ,Wahrheit/ entgegentreiben, vielmehr im Gegenteil. Vom Typus her haben wir heute ein tendenzielles Veralten, auch schlicht Banal-Werden, Nietzschescher Reflexionen, nietzschesch gebauter Theorie, zu gewärtigen und dafür Wirklichkeiten, die die Hegelsche Konzeption gerade immer moderner werden lassen.

Hegel-und-Nietzsche: das ist in der Tat die Entscheidung über das Wesen der Moderne. Von seinen Gegnern her läßt sich das Potential und die Begrenzung des Hegelschen Konzepts ausloten. In seinen Widersachern artikuliert sich, was die Erfahrungen der veränderten gesellschaftlichen Wirklichkeit an neuem zusätzlichem Problem-Druck, neuem Denk-Erfordernis geschaffen haben. Der mit dem Namen Nietzscher verbundene Protest gegen das Normative von Vernunft und Allgemeinheit, gegen gesellschaftlich-sittliche Formen und Institutionen und gegen die Ideale des modern Sozialen ist die Spitze davon.

Diese Entwicklung hat zugleich einen prinzipiellen, systematischen Aspekt in den Blick gehoben. Er soll hier, am Punkt der denkgeschichtlichen Entscheidung thematisiert, als die Frage der >moralischen Integration im Hintergrund stehen. Unter >moralischer Integration، soll, hinausgehend über die klassische soziologische Integrationsthematik von Durkheim und des Strukturfunktionalismus, ebenso die weiterführende Differenzierung des funktionalistischen Theorieparadigmas in 〉System- $<-v s .->$ Sozialintegration «, aber auch gegen Konzepte der >kulturellen Integration`, der Milieu-Integration, der LebensstilIntegration, die Subjektsseite moderner differenzierter, pluralistisch-komplexer Gesellschaftsverhältnisse verstanden werden. >Integration ist hier nicht primär an den Mechanismen gesellschaftlicher Ordnung festgemacht, als das Integriertsein/-werden der Aktoren in Prozesse und Funktions-Positionen, durch die Prägung auf konforme Muster sowie Internalisierung, und auch nicht modelliert nach Konzepten einer der Ordnung zugrundeliegenden, ideell-normativen Struktur, d.h. >Integration< als die normativen Geltungen und diese lebensweltlich geronnen in >gefühlten` Gemeinschafts->Identitäten smoralische Integration، will vielmehr nach den individuellen Erfahrungen mit gesellschaftlichen $\mathrm{Ab}$ - 
straktions-, Anders-seins- und 'Entfremdungs -Zumutungen fragen: den Bindungen und Verbindlichkeiten der Subjekte unter diesen Bedingungen. ${ }^{4}$

Integration - und die Stärke von Integration - gestaltet sich (und bewahrt sich!) so darin: daß die Subjekte, mit ihren persönlichen moralischen Empfindungen, sich als zugehörig, und als respektiert und anerkannt, zum betreffenden Allgemeinen erfahren bzw. erfahren können; daß keine Erfahrungen (oder doch ressentimenthafte Binnen-Meinungen) von prinzipiellem Diskriminiert-Werden sich aufschaukeln und verfestigen; daß (etablierte) Instanzen als 'gerecht< empfunden werden und stellvertretendes Agieren und Urteil - in meinem >Namen< - als berechtigt, Repräsentationen und Stellvertretungen statt meines eigenen entsprechenden Tätigwerdens; auch, daß die Einzelnen in einem weitgehenden >Sicherheits Gefühl leben (Gefühl der leiblichen und - jedenfalls gegebenheitsbesten - ökonomischen Sicherheit); daß gewisse Eingriffe aus shöheren Erforderlichkeiten oder Ziel-Überzeugungen, Eingriffe in meine lokale Lebenswelt und meine situativen Interessen (z.B. um des übergeordneten, auch Notfall-Schutzes, der >öffentlichen Ordnung ( oder der Zukunfts-Vorsorge willen), als hinnehmbar empfunden werden; überhaupt daß bestimmte über-individuelle - und gruppierungs-neutrale, parteien-transzendierende $>$ Prinzipien als akzeptiert verbreitet sind und als gültig behandelt werden, namentlich Entscheidungsund Verteilungs-Prinzipien; und schließlich entsprechend die Disposition bzw. Bereitschaften zur Eingliederung, Mitwirkung, und einen eingeforderten Beitrag zuzusteuern - habituelle Einstellungshaltungen, eigenen Vorteil, Originalagieren-in-eigener-Sache und Wert-Optionen des persönlichen Lebens bzw. Lebenswegs so weit trennen zu können ${ }^{5}$ von dem Aktuellen des größeren (Gemeinschafts-) Ganzen, daß ich nicht um deren Durchsetzung willen gegen das Bestehende agiere, wüte, grolle.

In all dem ist das Hegel-und-Nietzsche ein vielschichtiger denkerische Reflex der Moderne. Auch Nietzsche und der Nietzscheanismus haben dabei ihren sehr spezifischen historischen Index. Die Wirklichkeiten der Moderne leiten kritisches Denken inzwischen gerade zu Hegel zurück, und der Aufsatz möchte zeigen, warum dem so ist. - Ich beginne mit einer Eingrenzung, zweiseitigen Eingrenzung der Frontlinie der Entscheidung.

\section{Hegel und Nietzsche: die Antipoden eines in sich zerrissenen Zeitalters}

1. Hegels politische Philosophie als Symbol einer vergangenen Epoche zu deuten, Epoche des herrschaftlichen Staates, der moralischen Autorität der institutionalisierten Kirche und des Glaubens an die Vernunft und den großen Gang ihres Fortschritts - Hegel selbst hat dem Zweifel an ihm mehr als genug Nahrung gegeben. Wohl steht seine energische Verteidigung des >republikanischen Gedankens, daß die Kraft eines modernen Gemeinwesens aus dem vereinigten Willen seiner Bürger erwächst: daß ein jeder Einzelner sein wahres Interesse darin wiedererkennt und verwirklicht sieht; ${ }^{6}$ und steht sein Eintreten für: die Gleichheit der Staatsbürger (unbesehen von Religion, Herkunft und ethnischer Zugehörigkeit), ${ }^{7}$ für die Rationalität wie Durchsichtigkeit von Verwaltungsakten, Rechtsprechung und fiskalischen Festset-

4 >Moralische Integration tegration, kein Sachverhalt des Ja-oder-Nein bzw. Überkommen-oder-Nichtmehr (bes.: -Nichtmehrerforderlich). Das wären gerade Modelle der Ordnung, dächten allein vom Ganzen her: und die moralische Integration wäre mit nur deren vormoderner Weise - absolute Bindungen und für alles (bes. religiös), gemeinsame verwurzelnde Wert-Überzeugungen - identifiziert, und Moderne als das Schwinden oder der Verlust genau dieser geteilten Seinsbegründungs- und Sinn-Absolutheiten (und statt derer die Transformation in funktional äquivalente Koordinationen der situativen Interaktion, die die Integration des Ganzen gerade weil wert- und überzeugungsneutralisiert leisten).

5 Sowie komplementär: eine gewisse Flexibilität - Reflexivität, souveränes Drüberstehen -, einige Dinge des Lebens nicht mit (Verlangen nach) Erfüllung von sIdentitäts<-Erleben zu überfrachten; d.h. unbeschadet der Integration im generellen, bzw. nicht jede einzelne Konstellation und Erfahrung zum casus zu machen.

6 Vgl. Enzyklopädie der philosophischen Wissenschaften, $§ 475$ (einschließlich »Zusätze« aus den Vorlesungen).

7 Vgl. Grundlinien der Philosophie des Rechts, § 209. 
zungen - gegen jederlei behördliche Willkür -, für bürgerliche Öffentlichkeit und (institutionalisiert geregelte) politische Partizipation, für Unternehmungen staatlicher Wohlfahrt, ${ }^{8}$ für prinzipielle Gewaltenteilung ${ }^{9}$ und für das Recht auf Beschwerde sowie Amtsaufsicht, und nicht zuletzt für den Schutz von Leben, Würde und Privatheit, von Eigentum und persönlichen Rechtsgeschäften. Ebenso hat Hegel klarsichtig die Rolle der Arbeit und der Wirtschaft in der modernen Gesellschaft thematisiert und die damit einhergehenden Probleme der industriell-kapitalistischen Produktion, ${ }^{10}$ des ökonomischen Imperialismus, sowie der Verelendung, des Pauperismus und der Vermassung. ${ }^{1}$

Doch zugleich mutet Hegel, im Bewußtsein der prinzipiell erreichten Vernünftigkeit ${ }^{12}$ dieser Einrichtung, uns Späteren das Unannehmbare zu, weder einzelnen gesellschaftlichen Gruppierungen noch erst recht den Individuen, ja im Grunde nicht einmal der (bzw. einer) religiösen Gemeinschaft oder ihren Würdenträgern, ein »Recht« zuzubilligen - Recht der Einmischung, des Protests und des gewissen Ungehorsams -, wo es um die ratio des staatlichen Ganzen und seines Agierens im Horizont der Geschichte gehe. Vollends leicht gemacht hat Hegel es seinen Verächtern, ja auch jeder Demagogie der Kritik, durch seine Behandlung der »Moralität $\ll .{ }^{13}$ Gegen sie hat er, bei aller prinzipiellen Anerkennung der darin sich selbst erfassenden - zu Selbstbewußtsein gekommenen - und sich bekundenden Subjektivität, doch im Konkreten hoch fragwürdige Aburteilungen ihres Anspruchs vorgebracht: hier eine ontologische Argumentationskeule zu schwingen - gegen jederlei moralische Handlungs-Orientierung und moralisches Sich-zu-Wort-Melden einfach fundamentalistisch den ontologisch nicht ganz klaren Status des moralischen "Sollens« ins Feld zu führen (»ein ganzes Nest gedankenloser Widersprüche ${ }^{14}$ ); dann immer wieder die Denunziation der Betrachtungshaltung, die am geschichtlich-politischen Geschäft agierend Beteiligten moralisch zu beurteilen (bloße »Kammerdiener «-Perspektive); die Geißelung der »Moralität « wegen ihrer (zugegeben unbestreitbaren) Gefahr der Selbstgerechtigkeit; und schließlich die mit dem Stufenbau des `Systems gesetzte alleinseligmachende Subordination der Moralität, d.h. des moralischen Vermögens und seines Rechts zur Berücksichtigung, unter die objektiv bestehende sittliche Wirklichkeit - mit der Voraussetzung, daß innerhalb des Raums der (»vernünftigen«) sittlichen Entitäten keine unabgegoltenen Probleme bleiben (oder neu hereinbrechen können), die für die Stimme des Moralischen überhaupt ein Feld ihrer berechtigten, gar erforderlichen Reflexion und Einmischung geben. In allem erscheint das moralische Verständnis eines Tuns oder Zutuenden und das moralische Stellungnehmen - auch jede moralische Regung, die über den Rahmen smeines` Staates hinausgeht ${ }^{15}$ - als sekundäre Einstellung: insgesamt ein bloßes Meinen, Räsonieren und Sich-wichtig-machen-Wollen, manchmal wohlfeil und bequem, manchmal besserwisserisch und manchmal auch nur eine dünkelhafte, larmoyante oder verbitterte Klage, die sich mit dem Gang neuer Verhältnisse nicht versöhnen will.

8 Vgl. ebd., $\S 241 \mathrm{f}$.

9 Vgl. ebd., $\$ 269,272 / 273$.

$10 \mathrm{Vgl}$. ebd., § 182-201.

11 Insofern - all dieser Punkte wegen - zu Recht auch immer wieder die Verteidigungen, die das Moderne der Hegelschen Sicht der Gesellschaft und des Hegelschen Staates herausgestellt haben. Vgl. nur die großen Deutungen von J. RITTER, Hegel und die französische Revolution, Frankfurt/M. 1965; S. AVINERI, Hegel's Theory of the Modern State, Cambridge 1972.

12 Bzw. beim letzten Punkt (Imperialismus, Verelendung usw.): Bewußtsein des schlechterdings Unvermeidlichen solcher Kollateralfolgen.

13 Vgl. Phänomenologie des Geistes (Ausgabe von J. Hoffmeister, Hamburg 1952), 423-472; Grundlinien der Philosophie des Rechts, § 105-141.

14 Phänomenologie des Geistes, a.a.O., 434.

15 Das Hinausgehende eines shumanitären`, eines menschheitlichen, eines die Zukunft der menschlichen Zivilisation oder das ‘ökologischer Schicksal des Globus geltend machenden und auch eines das Zusammenleben der Völker betreffenden Kriteriums erscheint vielmehr als dahinter privatistisch Zurückbleiben. 
Hegels politisches Denken hat sich damit eine offene Flanke gegeben. Im einzelnen liberal, ${ }^{16}$ scheint die von Hegel gezeichnete politische Ordnung so im Ganzen doch manifest machtstaatlich: im Ganzen, d.h. beim faktischen modernen gesellschaftlichen Pluralismus - Pluralismus der Überzeugungen in bezug auf die höchsten Zwecke sowie Pluralismus der Lebensweisen und damit verbundenen ,Werte ‘-, bei der Frage der Legitimität und Frage des Monopols seiner Einrichtungen und Organe. Die Anschauung (inhaltlich wie theoriemethodisch), im Gefüge der Institutionen der Ordnung bleibe nichts, bei dem die moralische Kompetenz, auch moralische Courage und aktives moralisches Engagement, der Bürger als Orientierung, als Korrektiv oder als >Halt! \&-Ruf vonnöten sein könnte, hat ihm den Ruf, nicht mehr auf Augenhöhe mit der Wirklichkeit zu sein, angeheftet. Es ist die Hypothek eines prekären moralischen Vakuums.

2. Wenn in dieses Vakuum, das das Zeitalter zunächst mit großen ethischen Entwürfen, vor allem auch großem ethischem Pathos besetzte, zunehmend Nietzscheanische Gedanken sich ausbreiteten, so ist freilich auch da, auf der anderen Seite, das Zeit- und Generationenphänomen vom generellen - und bleibenden - Fortgang des Denkens zu unterscheiden und dieser wiederum von dem in diesem $>$ Nietzscheanismus $<$ damit transportierten Voraussetzungen. Auch in bezug auf den $\gg$ Nietzscheanismus $<$ der Epoche seit dem Ende des 19. Jhs. gibt es etwas, das getrost dem historischen Blick überlassen bleiben kann, als Mentalität einer bestimmten geschichtlichen und gesellschaftlichen Konstellation. Und gibt es vor allem etwas, was nicht das Problem ist, Problem der prinzipiellen Abwendung vom klassischen in Hegel verkörperten Denken. Auch für die Wirkungen Nietzsches gilt, daß man hier nichts unbesehen eins zu eins nehmen kann. Nichts ohne Differenzierung und historische Situierung.

Als in den letzten Jahren vor der Wende zum 20. Jahrhundert, und dann binnen kurzem in einer sich überschlagenden Bewegung, die Rezeption Nietzsches einsetzte, hat er von Anbeginn in vielfältigen Linien ins philosophische Denken hineingewirkt. Die bleibendste Aufnahme war die des Kulturkritikers und des Entlarvers - die Aufdeckung heimlicher dahinterstehender Motive in den so stolzen geistigen und gesellschaftlichen Errungenschaften der Zeit. ${ }^{17}$ Hier freilich steht die Gestalt $\gg$ Nietzsche keineswegs so singulär und erratisch, wie er sich selbst und vor allem dann auch seine Anhänger ihn stilisiert haben. In einem Klima von einerseits Ausweitung naturalistischer Erklärungen, Ausweitung eines swissenschaftlichen Weltbildes, und zugleich andererseits allgemeinem Klima, das Leben der Kultur und der IIdealer abzuspalten von den profanen (naturalen) Prozessen des Sozialen, war Nietzsche gar nicht so fern. Und auch in der wissenschaftlichen Profilform und Gewichtsetzungen konnte ein Nietzschesches Denken sich in einigem Wesentlichem durchaus in die Gegebenheiten der Zeit einfügen. Mit ihnen teilt es die Aufwertung von Prozessen eines Unbewußten (wie seit der Romantik und E. v. Hartmann); die nüchterne, unsentimentale Moraldekonstruktion (wie in empiristisch-sensualistischen oder utilitaristischen Richtungen); auch ein generelles 'genealogisches Denken (wie seit Hume in bezug auf Moralvorstellungen und Religion, seit Fichtes und Schellings 'Geschichte des Bewußtseins` oder Hegels `Phänomenologie des Geistes (); die zunehmend offene Kritik des Christentums, theologisch wie als bestimmenden kulturellen Faktors der Geschichte (wie seit D. Fr. Strauß); die Thematisierung von Sinneserfahrung und Leiblichkeit (wie seit Schopenhauer und Feuerbach); den Boom von 'Erkenntnistheorie،, von philosophischer (Wissenschafts-) Grundlegung als Erkenntnistheorie; die Bedeutung spsychologischer< Forschungen; sowie nicht zuletzt die Einsicht in den Konstruktions- und auch fiktionalen Charakter un-

16 Liberal insbes. angesichts der Realitäten seiner Zeit und angesichts der damaligen anderweitigen theoretischen Gesellschafts- und Staatskonstruktionen.

17 Die Rezeption Nietzschescher Gedanken ist inzwischen für alle großen nationalen Kulturtraditionen aufgearbeitet. Verwiesen sei hier nur auf die deutsche und die polnische: St.E. ASCHHEIM, Nietzsche und die Deutschen. Karriere eines Kults, Stuttgart / Weimar 1996; W. Kunicki (Hrsg.), Friedrich Nietzsche i pisarze polscy, Poznań 2002; G. Hübinger, A. Przyłębski (Hrsg.), Europäische Umwertungen / Europejskie przewartościowania, Frankfurt/M. 2007. Ferner die neue Dienstbarmachung im Denken der >Postmoderne $₫$ : vgl. A. RECKERMANN, Lesarten der Philosophie Nietzsches. Ihre Rezeption in Frankreich, Italien und der angelsächsischen Welt 1960-2000. Berlin / New York 2003. 
seres Wirklichkeits-Wissens (die wir auch von Avenarius über Poincaré bis in den Neukantianismus finden).

Für die Rezeption Nietzsches gibt es insofern eine wesentliche Schicht dieses Prozesses, in der sie vielfältig vorbereitet war. Gleiches gilt für das, was man den allgemeinen `Nietzscheanismus` des Zeitalters nennen kann. Dort ist vieles wohl nur äußerlich mit dem Namen Nietzsche, als die (übrigens oft nur indirekt oder vom Hörensagen bekannte) scheinbar radikalste Ausformung eines großen Drängens, verbunden. In einer Lage der zunehmenden Unübersichtlichkeit stellten sich die typischen Krisenphänomene ein: die Suche nach einem anderen, eigentlichen Zustand des Menschen, die Rücksichtslosigkeit gegen die Instanzen des Alten und zumal die herrschende Moral, sowie das Verlangen nach einer großen kulturellen Inventur, nach Klärung des wahrhaft Gültigen und Wertvollen.

All diese Aspekte eines Nietzscheanismus, sooft er sich dann im 20. Jh. noch einmal neu wiederholen sollte, sind eigentlich nicht die Herausforderung für Hegel, ja wohl nicht einmal ein wirklicher Gegensatz. Vergleichbares gab es auch zu anderen Zeiten, als Gegenströmungen gegen die großen, offiziellen Theoriegebäude der Philosophie, oder, mit Kant zu sprechen, als Protest des ,Weltbegriffs ‘ des Verlangens nach denkerischer Orientierung gegen den 'Schulbegriff^ der Philosophie. Das >Moralische، liegt hier in der Suche nach einer neuen >Weltanschauung«. Und die >philosophischen Thematisierungen dieses Nietzscheanismus, mit denen sein Denken sich gegen das vormalige der Tradition richtet, sich in Front bringt: angefangen beim ,Genealogischen ( und >Psychologischen (nd den >Leib -Aspekten, sind genauer besehen vor allem Ausweitungen des Frage-Horizonts. Sie ließen sich, soweit zu sehen, durchaus in Hegelschem Geist reformulieren, und integrieren. ${ }^{18}$

Wenn >Nietzsche zum Signum eines fundamentalen Epochenbruchs geworden ist, eines Bruchs, der mitten durch die Moderne hindurch geht - wenn `Nietzscheanismus` des Denkens zu mehr geworden ist als einem begrenzten zeitbedingten hype, einer bloßen Modeerscheinung oder Zeitgeist-Mentalität, so ist es darum nicht nur der kritische Gestus als solches. Von dem vielmehr ließe sich vieles gerade auch in Hegel finden; und viele der Thematisierungen würden auch zu einem im Sinne der neuen Einsichten des 19. Jhs. fortgeschriebenen, einem weiterentwickelten Hegel gehören. Damit dies zum wirklichen Entweder-Oder wurde und die Verdrängung, ja Überzeichnung Hegels sich aufspreizen konnte, mußte denn auch eine noch wenig gesehene andere Dimension Nietzscheschen Einflusses: etwas Konzeptionelles hinzukommen.

\section{Die Dekonstruktion des Sozialen}

So wie Nietzsche selbst seine anfängliche Gegenwartskritik der Unzeitgemäßen Betrachtungen und von Menschliches, Allzumenschliches zunehmend zum ganzen Theoriegefüge und philosophisch-generellen Argumentationen ausgebaut hat, so galt auch die Rezeption immer stärker dem in ihm verkörperten grundsätzlichen Typus des Denkens - und hier vor allem in einem sehr spezifischen Punkt. Die Rezeption galt mehr und mehr einem Wesensmerkmal Nietzscheanischen Denkens, das hinter den lauten - den bekannten - Topoi vom >Tod Gottes, Kleingeistigkeit (〉Bauernaufstand ) christlicher Ideale, ,Umwertung aller Werte`, Relativierung der Rationalität usw. sich leicht versteckt. Es ist die von Nietzsche exemplifizierte Fassung des Sozialen überhaupt. Sie erst ist es, was mit dem ganzen klassischen Erbe des Denkens bricht.

1. Denn was philosophisches Denken als >Kulturkritik heißt: zu den großen Gegnern der Nietzscheschen Kulturkritik gehören zentral auch die Formen des Gesellschaftlichen, und gehören das Selbstverständnis dabei, die Ideale der Zeit über das soziale Leben. Mit sich steigernder Radikalität springt die (bekannte) Moral-Kritik über auf eine Sozial-Kritik. Gesehen hat Nietzsche, daß Sozialverhältnisse, und gerade auch in dem habituellen Auf-etwas-eingestellt-sein und insofern Funktionieren, stets verschlungen sind mit Vorstellungen über sie, nicht zu trennen davon. Die Sprengkraft des Nietzscheschen Kriti-

18 Das zu leisten wäre freilich eine noch einmal ganz eigene Aufgabe. Auch ist dazu (von plakativen Parallelisierungen, die bei beiden etwa gerade das >Machtstaatliche (herausstreichen, abgesehen) bisher noch wenig vorbereitet. 
zismus speist sich wesentlich aus dieser Offenlegung des sozusagen sideologischen Unterbaus des sozialen Lebens. Dies macht, zusammen mit seinem feinen Sensorium für die - die rationalistischen und menschheitsbeglückenden - Denkformen in den im 19. Jh. entwickelten Modellen von Moralreform und Moralität-des-Sozialen, die Stärke des Nietzscheschen Zugangs aus: und sein bis heute anhaltendes Überzeugungspotential.

Doch diese Stärke geht einher mit zugleich der Schwäche des ganzen Argumentationskonzepts: nämlich alles Soziale dergestalt nur in solchen Kategorien zu bestimmen, auf solches zurückzuführen. Dabei wird obendrein Soziales, Sozialität wesentlich mit dem überhaupt identifiziert, was seit dem 18. Jh. als deren neue Wirklichkeit entstanden ist und was von Hegel auf den Begriff der »bürgerlichen Gesellschaft« gebracht worden ist. Alles Soziale - mit all ihren Allgemeinformen, Regeln, Funktionsmechanismen, Ideen - erscheint, vom Argumentationskonzept her, nur als Phänomen der Bürgerlichkeit dieser »bürgerlichen Gesellschaft «. ${ }^{19}$

'Nietzscheanismus` des Denkens: das ist insofern von vornherein und grundsätzlich eine (um der globalen Kritik, ,Kulturkritik،, willen betriebene) Nivellierung, ja Reduktion der Sachverhalte. Und, was daran problematisch ist, wird verschärft durch ein Zweites. Denn dies Umgehen mit Sachverhaltsausprägungen geht notwendig einher mit einer Kehrseite davon. Eine solche Universalisierung der Phänomene und ein solches Einheitsmodell der Kritik bedarf, um überhaupt eine kritische Spannungsdifferenz aufzumachen, nicht im Einerlei der perhorreszierenden Beschreibung zu verplätschern, unweigerlich eines Außenpols. Die >Kulturkritike, um überhaupt Argumentation zu sein, bedarf notwendig einer entsprechend prä- oder a-gesellschaftlichen - im Grunde: prä- oder a-sozialen - Dimension des menschlichen Bezugs.

Und dies ist bei Nietzsche die so ziemlich blendendste, aber prekärste Möglichkeit davon: nämlich eine an vor-modernen Sozialformen abgelesene Weise der direkten Person-Person-Beziehung. Alle soziale Wirklichkeit, alles soziale Phänomen wird auf der Folie eines wesenhaft vormodernen Zustands persönlicher Verhältnisse gesehen. Alle sachlichen Formen, und auch die zunehmend als `Entfremdung` erfahrenen Versachlichungen in der gesellschaftlichen Wirklichkeit, werden als bloße Degenerationsphänomene eines kraftlosen und nivellierten Soziallebens dechiffriert. Nietzsches Konstruktion ist, vielmehr Herrschaft - und komplementär Unterordnung, Einfügung - als sozialen Primärbegriff anzusetzen. Herrschaft, und basierend auf personalen Beziehungen, ist hier im ganzen als DimensionsKategorie alles Sozialen gesetzt: und direkt von Person-zu-Person - dem Anderen der Herr zu sein -, in einem Immediatismus des Hohen-und-Niedrigeren. ${ }^{20}$

2. Um keine falschen Frontstellungen aufzumachen: ${ }^{21}$ Diese Problematik schmälert nicht die vielen >soziologischen Einsichten, welche bei Nietzsche zu finden sind - Einsichten vor allem in das Nichtrei-

19 Abgelesen dabei zudem an zumal den deutsch-bismarckreichischen Ausprägungen davon.

20 Es geht in diesem Aufsatz um die Art des Nietzscheschen Denkens - und die Wirkungstradition dieser Art-, insofern sei ihm nachfolgend manches dünkelhaft Uninformierte, manches unerträglich Großsprecherische, Vom-hohen-Roß-herab-Beurteilen (der "psychologischen « Motive anderer) und manches schlicht Dumme nachgesehen. Abgesehen sei von all den Ressentiments, von denen genau auch die Nietzschesche Philosophie selber durchsetzt ist, und all den Idiosynkrasien. Dort hat die Art dieses Denkens einfach nur ihre persönliche Ausartung, und das tut im Grunde nichts zur Sache. Darunter etwa die Propagierung der sozialen »Kasten«-Ordnung oder Nietzsches Auslassungen zur "Arbeiter-Frage« oder die Tendenz zur Personalisierung sozialer Gedanken und Ideen, d.h. Inhalte, wenn sie von den (charakterlich) ,falschen Welt gebracht wurden), ebenfalls sofort und global als falsch anzusetzen (vgl. als nur zwei exemplarische Beispiele unter vielen: Der Antichrist, Aph. 57; Götzen-Dämmerung, Aph. 40). All solches mag als Mentalitäts- und Zeitphänomen - wenn nicht gar als biographisches Privatproblem - einfach dahingestellt bleiben; argumentativ jedenfalls kann man wohl nur schicklich darüber hinwegsehen.

21 Eine falsche Frontstellung wäre auch, beides gegeneinander auszuspielen - etwa daß >wahre ২ Sozialformen und -normen, jedenfalls modern, nichts wesentlich mit Herrschaft zu tun haben, nicht auch gerade Momente von Macht darin fungierten. 
ne, >Ideologische〈 sozialer Rationalität und in die (sozial-) psychologischen Mechanismen gesellschaftlichen Handelns. Doch, die von Nietzsche begründete Perspektive ist so im eigenen Konzept gänzlich einseitig. Erkenntnis des Sozialen - dieses ganzen Bereichs von Formen und Mechanismen und wirkenden Ursachen - kommt hier nur als Kritik der sozialen Ideale in den Blick, als Dekonstruktion, Entlarvung der unnatürlichen, sekundärentstandenen Vorurteile und Fiktionen des Soziallebens. Jede andere, nicht negativ-demaskierende Erkenntnisaussage, Erkenntnisgrundlage und Erkenntnisprogramm bzw. Methodenideal gilt damit lediglich als Teil des zu Verwerfenden selber: als Reproduktion, Verdopplung der herrschenden Illusionen und normativen Ideen. Alles, dominante Gedanken der Zeit wie auch etablierte Wissenschaft, erscheint nur als Abbild gerade der Verfallsform des Sozialen. Alles, was das Zeitalter als Errungenschaft kultiviert, wird zu im Gegenteil nur einer Folge des Niedergangs.

»Die [heute so hoch beschworene] >Gleichheit< [zum Beispiel - dahinter steht nur] eine gewisse thatsächliche Anähnlichung, die sich [vielmehr] in der Theorie von 'gleichen Rechten` nur zum Ausdruck bringt, [und diese Gleichheit] gehört wesentlich zum Niedergang: [sie setzt voraus, daß] die Kluft zwischen Mensch und Mensch, Stand und Stand, die Vielheit der Typen, der Wille, selbst zu sein, sich abzuheben [... - ], das, was ich Pathos der Distanz nenne [und was] jeder starken Zeit zu eigen [ist, geschwunden ist]. Die Spannkraft, die Spannweite zwischen den Extremen wird heute immer kleiner, - die Extreme selbst verwischen sich endlich bis zur Ähnlichkeit ... «"2

Und das aufgekommene Wissenschaftsprojekt einer »Sociologie«, als Analyse und Kritik des Gesellschaftlichen und Entwurf einer entsprechenden ('rationalenc) politischen Ordnung, habe die Kriterien und Ideale ihres Erkenntniswollens gerade mit ihrem Gegenstand gemein. Kriterien und Ideale der theoretischen ratio seien in allen wesentlichen Hinsichten nur am bestehenden Faktischen abgelesen.

"Alle unsre politischen Theorien und [auch real die] Staats-Verfassungen [...] sind Folgerungen, FolgeNothwendigkeiten des Niedergangs; die unbewusste Wirkung der décadence ist bis in die Ideale einzelner Wissenschaften hinein Herr geworden. Mein Einwand gegen die ganze Sociologie [...] bleibt, dass sie nur die Verfalls-Gebilde der Societät aus Erfahrung kennt und vollkommen unschuldig die eigenen VerfallsInstinkte als Norm des sociologischen Werturtheils nimmt. Das niedergehende Leben, die Abnahme aller organisierenden, das heisst trennenden, Klüfte aufreissenden, unter- und überordnenden Kraft formulirt sich in der Sociologie von heute zum Ideal .... ${ }^{23}$

Sämtliche geistigen und kulturellen Errungenschaften, worauf das Selbstverständnis der Zeit basiert, fallen damit der totalisierenden Entlarvungs-Strategie, ihrer globalen Umkehrung der Werthaltigkeitsperspektive, anheim. Mit dem Zeitalter teile auch der intellektuelle Diskurs darüber und gerade auch die rationale Wissenschaft denselben Mangel: das, daß etwas Nicht-Allgemeines nicht wahrhaft sein könne und daß Ungleich-Sein und Sich-Abheben nicht rechtfertigbar seien, als Voraussetzung und >moralisch< hochbesetzte Norm in sich zu transportieren. --

Hinter der Kritik an den zeitgängigen sozialen und kulturellen Idealen bringt sich darum in Wahrheit noch etwas Grundsätzlicheres zur Geltung. Die virulente sKulturkritikı, in ihrer Nietzscheanischen Radikalisierung, ist nicht so selbstevident und sharmlos`, wie sie in ihren Aufdeckungen erscheint. Die Offenkundigkeit ihrer Inhalte bzw. Thematisierungen verdeckt auch ein Stück massive Problematik ihres Argumentationstypus und Begründungen. In ihr ist, unterderhand, nichts weniger als eine ganze Dimension weggestrichen. Es ist eine gesamte Dimension weggestrichen in der Sache und weggestrichen in der (wissenschaftlichen) Erkenntnis- und Argumentationsaufschließung.

22 Fr. NIETZSCHE, Werke. Kritische Gesamtausgabe, hg. v. G. Colli / M. Montinari. Bd. VI,3, Berlin 1969, 132f. [Götzen-Dämmerung (1889), Aph. 37].

23 Ebd. Nietzsche bezieht dies auf die "Sociologie in England und Frankreich «: das heißt die szientistische Tradition davon. - Nichts zur Sache tut, wie viel er davon wirklich kannte. Sicher aber ist, daß er die zu seiner Zeit, v.a. in Deutschland, entstandene andere Tradition eines kritischen Sozialdenkens so gut wie nicht wahrgenommen hat. 


\section{Erpreßte Übermenschen (Der Erfahrungsgehalt der Nietzscheanischen Opposition)}

1. Bei allen berechtigten konkreten Beobachtungen, der Preis ist hoch. Das gesamte Beziehungsgeflecht eigentlicher ssozialer Prozeßzusammenhänge ist verschwunden, sowohl Prozesse des handfest Gesellschaftlichen als auch (generellere) >Intersubjektivitäts ‘-Prozesse. Bei Nietzsche ist ein blinder Fleck an der Stelle des eigentlichen sSozialen<: an der Stelle dessen, was definitiv seit Hegel an Einsichten gewonnen war - Einsichten auch in überhaupt die Eigenständigkeit, Eigengesetzlichkeit dieser mitspielenden Dimension - und was, d.h. welche »Geist«-Prozesse, seit der weiteren Wissenschaftsentwicklung des 19. Jahrhunderts zunehmend als materialiter Soziales und Gesellschaftsmechanismen dechiffriert, konkretisiert worden ist. Die Nietzscheanische Radikalisierung: das ist, hier vielmehr im ganzen einen blinden Fleck zu haben. ${ }^{24}$ Die >Kulturkritikı Nietzscheanischen Typs operiert damit, ja konstituiert sich nachgerade dadurch, bei all jenem strukturell >Sozialen ( einfach nur eine Leerstelle zu haben. Es ist, und eben ungeachtet der nicht wenigen eingeflochtenen tiefen Beobachtungen, eine Konstruktion, in der das >Soziale als ein Eigenes systematisch ausfällt: ausfällt in Phänomenbestimmung bzw. -Sensorium und ausfällt in der Argumentation. Bestimmte Faktoren, ganze Bereiche von Faktoren kommen, strukturell, gar nicht vor!

Statt dessen ist zumal alles modern Soziale wie auch entsprechende Wissenschaftsprogramme und deren Erkenntniskriterien oder -kategorien kurzerhand nur als Degenerationserscheinungen gesehen, Erscheinungen der Degeneration von wahrhaft kraftvollen, mit natürlichen Herrschens- und EhrfurchtsFormen verbundenen menschlichen Lebensbeziehungen. Alles erscheint, global, als bloßes Produkt geschwundener Kraft bzw. als Ausdruck von Ressentiment (und moralisch: als verschleierte Unaufrichtigkeit): vom Kriterium des intersubjektiv >Objektiven<; über den Begriff des >Rechts - bei Nietzsche ist >Recht< eigenartig nur als Rechtfertigung verstanden und als Vorrechte, Anrechte, Befugnisse; dann überhaupt Institutionen - Nietzsche kennt hier grundsätzlich nur Hierarchie-Institutionen (und dabei personale Autorität); ${ }^{25}$ den Raum der Öffentlichkeit - nur ein Andrängen und Sichbreitmachen des unberufenerweise bei allem Mitreden-Wollens, ja der geilen Frechheit des Pöbels; das `Menschenbild und die Ideale des humanen Mit-einander-Umgehens; bis schließlich hin auch zur Wissenschaft und rationalen Kritik. Alles, was dabei vom snatürlichen ' Autoritäts- und Vornehmheits-Empfinden abweicht, rückt in diesem Denken in dieselbe Rubrik mit (persönlichem) moralischem und mentalem Makel.

2. Ein immenser Verlust an Komplexität der Phänomensicht und Argumentationskomplexität jedenfalls. \Kulturkritik Nietzsches: das sind eben nicht nur die erhellenden Diagnosen, zumal für vieles davon man Nietzsche wohl nicht gebraucht hätte, auch ein weiterentwickelter Hegelianismus dies hätte sehen lassen. Sondern es ist auch bestimmt durch die mit Nietzsche etablierte Weise des Wie-erklärt, Wie-begründet dabei. Und dies fällt hinter den Stand eines ganzen Jahrhunderts zurück. - Weshalb dann konnte ein solches Denken, und bis heute mit immer neuer Infektionskraft, zur Wirkung kommen? Ich möchte behaupten: es ist die in ihm enthaltene >Wahrheit‘, nämlich unreflektierte Wahrheit. Denn die Nietzschesche Kulturkritik, der Typus ihrer Reflexionen, hat eine indirekte, eine in ihr selber gerade unerkannte Richtigkeit. Man darf dies den Erfahrungsgehalt dieses Denkens nennen.

Die Philosophie Nietzsches, und der Nietzscheanismus der Rezeption, ohne Zweifel hat ihren Erfahrungsgehalt. Doch das ist ineins ihr Problem gerade. Dieses Denken gehört seiner Zeit zu: aber zu unmittelbar. Was Nietzsche kritisiert, was er dagegensetzt, welches Gegen-Ideal, und wie er dabei argumentiert - es ist ein erfahrbar Gewordenes. Ein Denken zu entfalten, das Allgemeinaussagen - Verallgemeinerungen und Theoreme - formuliert, obzwar es sehr spezifisch seinen Zeitbezug an sich trägt, das betrifft nicht nur, auf der Seite der positiven Einsichten, seine Kritik der modernen Rationalität. Der Zeit zuzugehören betrifft nicht nur jenes Sensorium, das er mit der ganzen Strömung der zeitgenössischen

24 Die globale - zuweilen inflationäre - „Willen-zur-Macht«-Terminologie, Bestimmung der Phänomene nach Maßgabe von »Willen-zur-Macht«, ist da nur die Folgen(außen)seite.

25 Leitbegriff ist allenthalben der von: »Rang«. Gedacht ist alles als festfunktionierendes, empfindenswie verhaltenshabituelles Von-oben-nach-unten; jedes andere nur: zwischenmenschliche Gleichmacherei, »Sansculottismus«, Impertinenz der Nivellierung nach unten. 
Kulturkritik teilt: das Sensorium für das Ungenügen des wissenschaftlichen Positivismus, in dem die Suche nach angehäuften Fakten zur blinden, selbstbezüglichen Unternehmung, rastlosem Mehr geworden war und unter der Masse des zu Wissenden alles Ziel und Sinn zum Verdecken gebracht hatte; dann Sensorium für den technologisch vereinnahmten Rationalitätsbegriff; überhaupt für die (๖rationalistischen () Machbarkeits- und Beglückungsideen der Zeit sowie alle >Fortschritts-Ideologie; für die zum Fetisch erhobenen Beschwörungen des `Staates` und der >Kultivierung «, des (>bürgerlichen $)$ Kulturlebens; und nicht zuletzt das Sensorium für die aus der Restmasse der Religion zurückgebliebenen profanen Ersatz-Moralen und Ersatz-Transzendenzen. Sondern ein erfahrbar Gewordenes ist vor allem allgemeiner, daß erst die Krise des gesellschaftlichen Gefüges - angestammte Positionen, Rollenfestlegungen und Lebensläufe - und der sozialen Bindungen, ferner das Auseinanderwachsen von verfachlichter Wissenschaft und demgegenüber Kultur bzw. Religion, die Separierung von söffentlichen` und sprivaten Lebensbereichen, von >öffentlichen` Räumen und familialer Intimität, ${ }^{26}$ sowie schließlich die Diskrepanz von Nationalkirche und persönlicher Spiritualität - daß überhaupt erst diese erfahrbaren Entwicklungen jenem Akzent auf dem Problem, ein Individuum zu sein, dem Pathos des authentischen/krafthabenden Individuums seine alles sich unterordnende Bedeutung gegeben haben. Nur wenn, angesichts von entstandenen Offenheiten, Ambiguitäten, Ungesichertheiten, vor Augen gestellten Möglichkeits-Visionen und gleichzeitig von realen Verhaltenszumutungen, die Kriterien persönlicher Identität, Kriterien meines Wer, nicht mehr selbstverständlich sind, bekommen die unthematisierten Voraussetzungen, aus denen die Nietzscheanische `Kulturkritik`, Phänomenbestimmungen und Theoriestruktur dabei, sich aufbauen, einen Resonanzraum. ${ }^{27}$

Die Welt ist so - ist es gewordener Weise -, wie die Philosophie Nietzsches und der Nietzscheanismus seiner Wirkung dies als Gegenfolie zeichnen: Das gesellschaftliche Leben vollzieht sich real nach Mechanismen des Willens-zur-Macht, zeigt $\operatorname{sich}^{28}$ als Prozesse, in denen Pole eines Willens-zur-Macht gegeneinander agieren. Ein den herrschenden Arbeitsformen und Formen des gesellschaftlichen Austauschs konformes Funktionieren wird zunehmend durch einschneidende gesellschaftliche wie ökonomische Sanktionen auf Schemaabweichung, Individualität, Eigensinn erzwungen. Integration und Identität sind massiv verbunden mit Zumutungen der Nivellierung, Zumutungen des gleichartigen Situationsverhaltens und der Werteanpassung. Industrielle Arbeit und Landflucht haben die Massen - die als Massen agierenden Gruppen - erst geschaffen, gegen deren Faktum das Nietzscheanische Denken >kulturkritisch sein Eigenes, seine normativen Gegenbilder aufrichtet; die Viel-zu-vielen der städtischen Massen sind dies, jedenfalls primär, sozusagen nicht aus freien Stücken oder individualcharakterlicher Neigung dazu. Die Rahmenvorgegebenheiten und Restriktionen der realen Lebens- wie Arbeitsverhältnisse erlauben immer weniger den Entwurf eines Über-sich-hinaus. ${ }^{29}$ Und überhaupt: das eigentliche Soziale ist zunehmend unsichtbar.

Darum: Nietzsche hat recht - aber aus anderen, im Endeffekt geradezu den gegenteiligen, als den in seinen `Entlarvungs ‘- und >genealogischen` Erklärungen aufgefahrenen Gründen. Es sind Gründe, die genauer: deren ganzes Feld - er mit seiner Argumentation genau aus dem Blick gebracht hat. Was er >kulturkritisch` diagnostiziert, hat eine massive Konstruktionshypothek: nämlich wovon dies Denken

26 Eingeschlossen die privaten Räume in (bürgerlichen) >Vereins ‘-Zusammenschlüssen.

$27 \mathrm{Zu}$ einer genaueren theoretischen und epochengeschichtlichen Erörterung vgl. R. ADOLPHI, »Gemeinschafts-Pathos und individualistische Fluchten. Der >Geist der Nietzsche-Zeit< und seine Kritik bei Helmuth Plessner«, in: Nietzsche und Schopenhauer. Rezeptionsphänomene der Wendezeiten, hg. v. W. Kunicki / M. Kopij, Leipzig 2006, 47-80.

28 Das heißt: was als offene Akteure erkennbar; doch selbst wo nicht, steht dies als erfolgreiche - und anerkannte - Maxime der Situationsorientierung und Verhaltensdeutung der Unterstellung solcherart, des Verdachts.

29 Schließlich auch: Herrschende Normierungs- und Konformitätszwänge greifen inzwischen so tief in die individuellen Lebensräume ein, daß es dort zu einer erpreßten Einschnürung auf ästhetische Kompensationen kommt - jene hilflosen `Musensöhne`, die dies Denken dann kulturkritisch als >Bildungsphilister $<$ geißelt. 
nicht spricht - nicht sprechen kann; bzw. welche Alternativen es systematisch zum Verschwinden bringt, eingeschmolzen hat. In der Evidenz der Erfahrungen, aus der sich seine Phänomenbestimmungen und die gezeichnete Perspektive speisen, ist der eine Reflexionsschritt weiter: nämlich zu der Gewordenheit dieser Zeiterfahrungen und zu den durchaus handfesten realen Faktoren dabei, nicht vollzogen.

In der Tat, die Gesellschaft - das soziale Leben und die kulturell-moralischen Normierungen des Verhaltens - ist unvertraut. Und die Verhaltensweisen gleichen sich an, die Verschiedenheit wie Distanz der Stände verschwimmt, die Schichtung ist flacher geworden. Doch dies ist bei Nietzsche genau als Prozeß der Gesellschaft selber unkenntlich gemacht. Verflochten ins Netz der harten Faktizitäten des gesellschaftlichen Systems, vermeint der Einzelne, sein Leiden sei metaphysisch - statt es als wesentlich auch gesellschaftliches Symptom, gesellschaftlichen Effekt sehen zu können: Und die Nietzschesche psychologisierende `Entlarvung reproduziert diese individualistisch-punktuelle Wahrnehmungsperspektive, die reine Binnenerfahrung des affektierten Individuums einfach nur, lediglich unter Hinzufügung einer Vertauschung der Vorzeichen, einer entworfenen Gegenmöglichkeit als dem gewertet Eigentlichen.

Es ist so, wie Nietzsche das modern Gesellschaftliche und den Habitus des daran Teilnehmens beschreibt. Aber es sind keine individualcharakterlichen, moralischen Ursachen, persönliche Ermattung, und anderes, gar Gründe des Gesellschaftsprozesses kennt die Nietzschesche Konstruktion nicht. Nietzsche: das ist der unreflektierte Spiegel bloß, Spiegel seiner Zeit.

\section{Versuchungen (Die Entsorgung der Gesellschaftstheorie durch >Kulturkritiks)}

Hegel und Nietzsche: Es gilt heute, bewußt diese totalisierende Provokation, die mit dem Namen >Nietzsche verbunden ist, in die Bemühungen um Hegel einzubringen. Die Nietzscheanische Provokation sie tangiert wesentlich auch die Gesellschafts- und politische Philosophie. Und dies, wie gesehen, in einer weitaus konkreteren Weise, als es in den bekannten Titeln wie 'Nihilismusı, >Umwertung ‘, Rationalitätskritik u.ä. gefaßt ist. ${ }^{30}$

In Nietzsche vielmehr kristallisieren sich die Motive, die die Abwendung der nachhegelschen Epoche von der Tradition des klassischen politischen Denkens bestimmt haben, die geistigen Kampffronten eines ganzen seitherigen Zeitalters. Nietzsche zeigt die Kehrseite des Prozesses der gesellschaftlichen Moderne. Die Kehrseite: angefangen bei den Phänomenen der Vermassung, und der Nivellierung der Lebensweisen; dem angstvollen Sicherheits-Denken und dem >Rechts ‘-Bedürfnis; der Furcht vor dem (nicht kontrollierbaren) Ausscheren, ja Anders-sein des Nebenmanns; dem eigenen Nichts-wagenWollen und nicht mehr persönlich für etwas Einstehen-Wollen; dem Sich-Verstecken hinter >Tatsachen', ,Wissenschaft « und >Fortschritt`; dem Mythos des ıStaates` und der Nation (nationaler Identität); und schließlich auch all den entsprechenden Rationalitäts-Vorstellungen, Rationalitäts-Ideen. `Nietzscheく wurde denn immer wieder zum Stachel des Denkens - Stachel auch gegen Ideen von Ordnung, Gerechtigkeit, Allgemeinheit, >Gesellschaftliches Leben`, ja überhaupt von Öffentlichkeit und `Vernünftigkeit`.

Gerade Nietzsche aber ist, so zeigte sich, in den entscheidenden Punkten denkbar unkritisch. Fahrlässig unkritisch, und aus zwangsläufigen argumentativen Gründen. Unkritisch bei den benannten Ursachen und unkritisch beim eigenen Gegenmodell menschlicher Bezüge und ihrer spolitischen، Formen. Bei allem, was Nietzsche teilt mit den Denkentwicklungen der nachhegelschen Epoche, ${ }^{31}$ eine entscheidende Tradition des 19. Jhs.: die gesellschaftstheoretische, nämlich wie sie von Hegel ausgegangen ist, Hegelsche Inspirationen den Erfahrungen entsprechend weiterentwickelt hat, hat Nietzsche gerade nicht aufgenommen. Und hat damit seine ganze Konstruktion, und auch die Diagnosen, in eine Schieflage gebracht. Was er von den das Jahrhundert umtreibenden genuin gesellschaftlichen Fragen überhaupt wahrgenommen hat, hat er in pauschaler Weise moralisch, wegen ihres unheroischen, sunmännlichen« Men-

30 Und im übrigen auch keineswegs nur wegen des Themas >Macht<, wo - wie vielfach zur großen Deutung, vor allem Epochendeutung erhoben - Hegel und Nietzsche auf dieselbe Seite träten, in dieselbe Schublade gepackt wurden.

31 Siehe oben Abschnitt II, 2. 
schenbildes, der Verächtlichkeit preisgegeben. In einem Zustand hoch komplexer GesellschaftlichkeitsProzesse hat der Nietzscheanismus das Soziale und dessen Formen einfach nur: re-individualisiert.

Wenn wir darum den Weg von Nietzsche zu Hegel zurück müssen, ${ }^{32}$ dann sollten wir es uns freilich nicht zu leicht machen. Das Problematische der Hegelschen Behandlung der Moralität bleibt. Daran ist nichts zu deuteln. Hegel mutet uns, neben all der Häme, ja Invektiven gegen das auf seine moralischen Überzeugungen pochende Individuum, nicht nur zu, keine explizite `Ethik $\mathrm{zu}$ haben, sondern auch, daß er die rethik ‘-haltigen Begriffe des Politischen, wie Gerechtigkeit, Legitimität usw., umdefiniert. Auf der anderen Seite Nietzscher ist in vielem etwas Grundsätzlicheres, als seine zeitgenössische Rezeption schon erkennen läßt. Man kann die Erfahrungen, aus denen die Motivationen, die auf Nietzsche und den immer neuen Nietzscheanismus führen, sich gespeist haben, lokalisieren in der aufgebrochenen mangelnden Integrationskraft des Sozialen: daß die sozialen Bindungen, Orientierungen und Normgebungen nicht Schritt zu halten vermochten mit den gesellschaftlichen Veränderungen der Epoche; und daß die vom klassischen politischen Denken, zuhöchst von Hegel angesetzten vermittelnden Institutionen ihre Funktionsaufgaben nicht mehr leisteten oder überhaupt zunehmend ausfielen. In der Tat mußte dem späteren 19. Jh. Hegels Denken mehr und mehr als lediglich eine Verteidigung autoritärer Ordnungsstrukturen erscheinen. Die Entwicklung hat die Hegelsche Konstruktion, ihre inhaltlichen Festlegungen, zum Bild der schlechten Wirklichkeit werden lassen.

Doch wo im skulturkritischen` Ankämpfen gegen dies das Nietzschesche Denken alle bestehenden Errungenschaften: Kultur wie soziale Formen und (norm-) geltende Einrichtungen, als lediglich Produkte derselben einfach schwach gewordenen Generation - in allem nur dieselben Ideale, Blendungen und Wunschbilder - sehen will, muß in vielem das Moralische - das sich-charaktertiefes-Individuum .... und das Gegenüberstehend-Personale und überhaupt das Vornehmheits-Gewahrende - für ein gesellschaftstheoretisches Problem einstehen. Angesichts dessen erscheint Hegels Einsicht, daß es in den gesellschaftlichen Strukturen Räume gibt und Räume geben muß, in denen nicht das Moralisch-sein-Müssen totalisierende Norm ist, Räume, in denen das bourgeois-Sein erlaubt, ja notwendig ist, umso moderner.

Hegels Kritik des moralischen Urteils, moralischen Einspruchs ist denn auch nicht so eindimensional und staatsvergottend, ${ }^{33}$ wie es eine nachfolgende Epoche verstanden und gezeichnet hat, evtl. auch verstehen mußte. Den Privatismus einer moralischen Konstruktion aller Verhältnisse vor Augen, Rigorismus eines moralischen $>$ Müßte eigentlich ...<, das Pochen auf $>$ muß mit meinen Überzeugungen vereinbar sein ${ }^{34}$ die Subjektivität, soziale `Institutionen` (im weitesten Sinne) von jedermanns Dafürhalten und Wertreflexion abhängig zu machen, und überhaupt den Purismus der strikten RechtfertigungsBegründung und Einsehens-Demonstration - all diese zwiespältigen Ausprägungen seiner Zeit haben Hegel die Rolle, was Moralität sein könnte und müßte unter Bedingungen moderner Gesellschaftswirklichkeit, sicher nicht so viel Aufmerksamkeit, Tiefe der Ausführung schenken lassen, wie es nötig gewesen wäre, aber auch wie sein konzeptioneller Ansatz es gekonnt hätte. Daß er nicht, genauer: wenig $-z u$ wenig -, von der positiven Funktion, auch Integrations-Bedeutung, der Moralität der Subjekte spricht, ist zum Großteil seinen konkreten Zeitumständen geschuldet: der Art der Besetzung des Begriffs durch seine Zeitgenossen, der sich verselbständigenden, verabsolutierenden Moralitäts-Fixierung und MoralitätsPathos. ${ }^{35}$ Und auch das aus heutiger Sicht offenkundig Illiberale in seinen Bestimmungen ist weithin ein

32 Ich möchte diese Folgerung hier zunächst einmal auf die in diesem Aufsatz behandelte Frage des Gesellschafts- und politischen Denkens - dessen konzeptionelle Struktur - beschränken und die Linien nicht auf die ganze Breite des einst von Löwith (s.o.) angesprochenen generellen Umbruchs ausziehen.

33 Bzw.: geschichtsgang-verabsolutierend - den faktischen Gang und das Sich-durchgesetzt-habendHerrschende als letztentscheidendes Urteil über das `Zu-recht « ( $»$ Die Weltgeschichte ist das Weltgericht«) und als Vorsehung.

34 Auch die Verschränkung von (vor sich hergetragen) Moralischem und Völkisch-Nationalem, wie bei nicht wenigen der Burschenschaftler.

35 Mir scheinen hier zwei Konsequenzen der rechten Interpretation zu ziehen zu sein: Man sollte nicht alles, was Hegel jemals bzw. irgendwo gegen die moralische Reflexion gesagt hat, - auch, was er 
Relations-Produkt, erwächst weithin aus den Veränderungen der Wirklichkeit, die die von Hegel gezeichneten (gemeinwesens-) institutionalistischen Lösungen überfordern und vielmehr gerade eines gewissen moralischen Korrektivs, moralischen Einmischung und >Halt!‘-Rufs der Bürger, auch schlicht der Anerkennung und Erfahrbarkeit des Beteiligtseins, bedürfen.

Was die Konzeption betrifft, so ist Hegel selbstredend nicht die Zurückweisung, ja Unterdrückung der Subjektivität aus Gründen des ordo. Hegels Denken vielmehr, sofern man nicht dessen eigene, eingeschränkte Terminologie zum Maß nimmt - abbildet -, sondern >Moralitätı der Subjekte allgemein faßt als in intrinsischen Überzeugungen, Wertempfindungen, Gerechtigkeits-Vorstellungen verwurzelte normative Reglementierungen von Verhalten und Urteil, sieht - und berücksichtigt theoretisch - mehrere

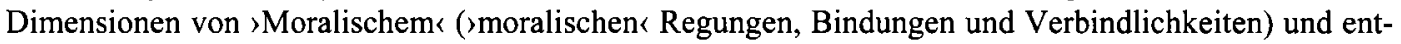
sprechend Integrations-Prozessen: elementare emotionale Zusammengehörigkeiten des mit einander Lebens und Wachsens, Erfahrung einer natürlichen Einheit, durchaus in anthropologischen und sozialanthropologischen Gegebenheiten verankert (Erfahrungen von >Familien modern Kleinfamilie) - das >Moralische` in Pietät, Liebe, Sorge, `Geschwisterlichkeit` und Erziehung (dem Anderen Kenntnisse, Fähigkeiten und `Haltung`zu zeigen); die Verbindlichkeit `öffentlicher`, d.h. meine unmittelbaren lebensweltlichen persönlichen Bezüge, Bindungen und erfahrenen Interessen übersteigender, Standards, Regeln, Positionen und Instanzen (Hegels »Sittlichkeits «-Stukturen von Gesellschaft und Staatsleben); dann das `Moralische $\prec$ als der (>innerliche $<$ ) Ich-Kern im Gefüge dessen, worin ich in meinem Handeln und Leiden verwoben, ggf. verstrickt bin - das `Moralische $<$ als >ich wilk, >ich rechne mir zu (anerkenne als mir zuzurechnen)^, sich verantworte«, sich finde mich darin wieder «, >ich erfahre meine Erfüllung usw. (im wesentlichen die Thematisierung der »Moralität « in der Philosophie des Recht $^{36}$ ); das >Moralischer als das in religiös-kulturellen Traditionen und >Weltbildern ( implizierte betreffende Normative, bzw. das daraus fließt - religiös-kulturelle Muster (Bilder) von Selbst und Selbst-Verfehlung, Selbst-Verlust, von Schein-Leben (larvenhaftem Sein) und Ich-Scham (das >Moralische in den resp. durch die Gestaltungen des »absoluten Geistes«); sowie schließlich das >moralische Beurteilen von allem und jedem als Hypertrophie des ent-wurzelten, sich aufspreizenden Subjektivismus - denn natürlich mußte nicht erst Nietzsche dem Denken die Degenerationen des modern Moralischen zeigen, auch Hegel kennt die Kritik an der falschen Subjektivität, an Selbstgerechtigkeit, wohlfeilem Moralisieren usw.

Gegen dies hat das Denken des weiteren 19. - und 20. - Jahrhunderts die Bewandtnisse von Moralischem vor allem wieder gleichgeschaltet. Der Geist der Zeit und die Abwendung von der klassischen Tradition und speziell dem Hegelschen Modell haben >Moralität! - das Postulat der (gesellschaftlich ermöglichten) rechten Moralität - zur Generallösung der menschlichen Welt aufsteigen lassen. Dabei freilich in einem konstitutiven Schwanken zwischen persönlicher Moralität, als individueller charakterli-

überhaupt unter dem Titel (Überschrift) »Moralität« führt - unbesehen durcheinandergehen lassen. Die Kontexte sind bei weitem zu verschieden. Und zweitens, es ist eher nur eine Verunklarung, Hegels Unterscheidung-Kontrastierung von »Moralität« und "Sittlichkeit« zu fassen als: Subjektivität vs. >Intersubjektivität‘, oder als: Prinzipienformalismus vs. >Gutes Leben،. Das bringt im Zweifelsfall nur Schematisierungen; außerdem stünde die positiv-übergeordnete Seite insgeheim von vornherein fest (wer kann schon gegen >Intersubjektivität` sein oder gegen das >Gute Leben`?!); und es überzeichnet die Hegelsche Theorie mit doch anders gelagerten denkgeschichtlichen Großpolaritäten, mit einem Kant-vs.-Aristoteles-Gegensatz oder mit einem Locke-vs.-Rousseau-, Individualitätvs.-volonté-générale-Gegensatz, ja führte den Verweis auf Hegels >Griechentum` wie eine alleserklärende starre Floskel mit sich. Im übrigen sind es ja alles strikt dichotomische Erklärungsschemata, was Hegels Theorie in bezug auf "Moralität « bewußt genau nicht hat.

36 Die drei dort thematisierten Problem-Polaritäten - Vorsatz und Schuld; Absichts-Anstrengungen und erlangtes Wohl; Ziel-Gutes und subjektives Gewissen dabei - definieren den Raum dieser >moralischen (Dimension (Grundlinien der Philosophie des Rechts, \$\$ 114-140; auch als Reformulierung der Problemstruktur der von Kant unterschiedenen dreierlei ,Normativitäten (im Praktischen, dreierlei Typus von »Imperativen«: Grundlegung zur Metaphysik der Sitten, BA 39ff.). 
cher Gesinntheit und in Horizonten direkt erfahrbarer Situativität, zuletzt Ich-Du-Verhältnissen, und andererseits moralischem Habitus qua Gruppen-Orientierung, Loyalitäts- und Solidar-Handeln, Überwindung bzw. Hintanstellung jedweden Individualismus: im einen, so der Weg Kierkegaards und auch Nietzsches, die Sozialverhältnisse - Gesellschaft, Institutionen, Positionen, Traditionen, herrschende Moral, aber auch öffentliche Religion - als nur noch repressives Diktat, als entfremdend und als Herrschaft des >Man< erfahren und im Gegenstoß gegen dies die Feier, ja Fanatik der Authentizität, Unbedingtheit des 'Selbst gegen die atomisierenden und egoistischen Verformungen, die mit moderner Gesellschaftlichkeit einhergehen. Beides hat von seinem Ethischen aus das Gesellschaftliche, in seinen Eigen-Prozessen, Struktur-Evolution, einschneidendem Modernitäts-Wandel und dann auch Institutionenform des Staats, wieder eingeschmolzen. ${ }^{37}$ Der philosophische Diskurs der ,Werte $<$, der seit Ende des 19. Jhs. die Motivationen der >Kulturkritik in sich aufgesogen hat und wie er der Boden wurde, der die immer neue Nietzsche-Rezeption innerhalb der Theorie vermittelte, hat - selbst wo die Begrifflichkeit der >Werte<, weil scheinbar kontaminiert, zwischenzeitlich teils wieder zurücktrat - dies dann theoriestrukturell verabsolutiert. Von der mehrfachen, komplex verwobenen moralischen Integration der Subjekte, die Hegels Theorie zu thematisieren eröffnet hatte, bleibt da kaum etwas übrig. Integration erscheint nur noch als Entweder-Oder eines generellen Typus, vor allem als der Weg von einer Totalbestimmtheit allen Lebens und Zusammenlebens durch >Weltbild -Werte zur funktionalen Differenzierung, die den gesellschaftlichen Zusammenhang scheinbar zunehmend neutral werden läßt in bezug auf die Moralen der Individuen, die subjektive Moral-Seite, scheinbar nicht mehr angewiesen ist auf moralische Integration.

Nietzscheanismus des Denkens hat insofern einen weiteren und zugleich engeren Hintergrund als das, wie er geschichtlich auftritt. Der Nietzscheanismus, seine Berechtigung, ist zu läutern von der vordergründigen Emphase der großen Zeiten-Wende. Nietzsche vielmehr: ist eine Versuchung. Und solange die klassische Tradition, solange Hegel nicht weitergedacht wird: bleibt eine betörende Versuchung. Das Zeitphänomen >Nietzsche` manifestiert, was das klassische politische Denken noch leisten mußte; und was zumal Hegel durch seine Behandlung des Moralischen zu einer irrlichternden EpochenPolarisierung hat zuspitzen lassen. Im Nietzscheanismus, in diesem Faktum der nachhegelschen Denkgeschichte bekundet sich, um welchen Schritt das Problem des Politischen komplexer, als bei Hegel explizit theorieausgeführt, ist. Es ist ein in vielem bis heute noch ausstehender Schritt. >Nietzsche ist nur die Kehrseite eines in der klassischen Tradition und bei Hegel noch nicht vollständig Geleisteten.

37 Wie Hegel demgegenüber gesehen hatte, sind die mehreren verschiedenen Dimensionen von Moralischem, deren menschliche wie normative Bewandtnis, im Hinblick auf die Dynamik und den Wandel der gesellschaftlichen Verhältnisse keineswegs irgend parallele Probleme (Probleme auch der Integration): In manchem wird die Integration als moralische in der Tat mehr und mehr zu einem Randphänomen; die Integration vollzieht sich dafür über - gelernt-internalisierte - abstrahierende Formalprinzipien der Interaktion bzw. Interaktionsbeteiligung. In anderem werden die Prozesse moralischer Integration hingegen umso nötiger (und auch erfahrbarer). - Ein sprechendes Beispiel für das Phänomensensorium, das gerade Hegels Theorie verkörpert, sind seine Überlegungen zur Solidarität mit den Armen (heutigentags thematisiert, würde dies den ganzen Bereich von 'Sozialtransfers` umfassen). Eine gut funktionierende Gesellschaft zeichne in ihrer modernen Entwicklung sich gerade dadurch aus, daß sie dies weitgehend vom Öffentlichen her, dem Gemeinwesen, übernimmt: und Moralität hier in Teilen entbehrlich macht. Von den Subjekten hier individuell Moralität einzufordern bzw. dies davon abhängig zu machen überforderte den Einzelnen auch, d.h. unter Bedingungen komplexer, in vielem auch indirekter Lebensverhältnisse. Es wäre eine Normativität, die das Menschliche der Erfahrungsräume weit überstiege; Erforderlichkeit und subjektive Erfahrbarkeit vielmehr haben sich zunehmend auseinanderentwickelt - und es kann insofern kein (hauptsächlicher) Sachverhalt persönlicher Moralität mehr sein; vielleicht einstellungs- und bewertungsmäßig noch, aber nicht in der wirklichen Tat. Vgl. Grundlinien der Philosophie des Rechts, § 242. 


\section{Rainer Adolphi}

Technische Universität Berlin

Institut für Philosophie (H 72)

Straße des 17. Juni 135

D-10623 Berlin

Email: rainer.adolphi@tu-berlin.de 